\title{
Distribution and characterization of Listeria monocytogenes clinical isolates in Portugal, 1994-2007
}

\author{
G. Almeida • A. Morvan • R. Magalhães • I. Santos • \\ T. Hogg $\cdot$ A. Leclercq $\cdot$ P. Teixeira $\cdot$ Research Team
}

\begin{abstract}
In recent years, the number of cases of listeriosis has increased worldwide. Ninety-five isolates of Listeria monocytogenes recovered from Portuguese human cases of listeriosis have been characterized by biotyping (cadmium and arsenic sensitivity), polymerase chain reaction (PCR) grouping, and by pulsed-field gel electrophoresis (PFGE) applying the enzymes $A s c \mathrm{I}$ and $A p a \mathrm{I}$. Isolates were classified into one of three PCR groups; IVb (71.6\%), IIb $(17.9 \%)$, and IIa (10.5\%). Four biotypes were differentiated: sensitive to arsenic/cadmium (48.4\%), arsenic-sensitive and cadmium-resistant $(25.3 \%)$, resistant to arsenic and sensitive to cadmium (18.9\%), and resistant to both heavy metals (7.4\%). Combined analyses of $A s c \mathrm{I}$ and ApaI patterns yielded a total of 58 PFGE types with five sets $(\mathrm{G}, \mathrm{Jb}, \mathrm{KKa}, \mathrm{Me}$, and $\mathrm{U})$ of Portuguese strains, each of which were indistinguishable by PFGE typing. In the present study, it was demonstrated that there are recurrent pulsotypes and that some were the same pulsotypes linked to outbreaks in France. In addition, there are some pulsotypes spread throughout the country, while others only appear in a restricted region. This study allowed the assembly of a first large pulsotype database of Portuguese clinical strains.
\end{abstract}

G. Almeida $\cdot$ R. Magalhães $\cdot$ I. Santos $\cdot$ T. Hogg $・$ P. Teixeira $(\bowtie)$ Centro de Biotecnologia e Química Fina, Escola Superior de Biotecnologia, Universidade Católica Portuguesa, Rua Dr António Bernardino de Almeida, 4200-072 Porto, Portugal

e-mail: pcteixeira@esb.ucp.pt

A. Morvan · A. Leclercq

Laboratoire des Listeria, Centre National de Référence des Listeria et Centre Collaborateur de l'Organisation Mondiale de la Santé pour la Listériose d'Origine Alimentaire, Institut Pasteur, 25, Rue du Docteur-Roux,

75724 Paris cedex 15, France

\section{Introduction}

Listeria monocytogenes is recognized as a major, opportunistic, foodborne pathogen in humans. The condition which this species causes, listeriosis, occurs worldwide both sporadically and epidemically, and has a case-fatality rate between 20 and $30 \%$. L. monocytogenes has the ability to cross the intestinal, blood-brain, and fetoplacental barriers, and, thus, septicemia, central nervous system (CNS) infections, miscarriages, and stillbirths are risks to immunodeficient or pregnant individuals [1].

In Portugal, listeriosis is not a notifiable disease and has, thus, been underestimated. Nevertheless, Almeida et al. in 2006 [2], in a retrospective study, reported an estimated incidence of at least 1.4 cases per million inhabitants in 2003. The implementation of an active surveillance program in the Netherlands led to a $43 \%$ increase in the reported incidence of listeriosis in the first year of operation [3]. This might give some indication of the current degree of underestimation in Portugal. Any such program would certainly omit reporting some sporadic cases and spontaneous miscarriages, as these are often not investigated. Since 2005, several European countries reported an increase of listeriosis and several foodborne outbreaks have been reported [4]. Some examples are the outbreaks which occurred in Switzerland associated with Tome cheese [5], in the United Kingdom associated with sandwich consumption [6], in the Czech Republic associated with soft cheese [7], and a recent multinational outbreak due to the consumption of "Quargel" cheese in Austria and Germany [8].

The characterization of $L$. monocytogenes strains can be based on its molecular subtyping by polymerase chain reaction (PCR) grouping or geno-serotyping [9] and by macrorestriction pulsotype analysis of its DNA using the 
PulseNet protocol [10]. The use of subtyping methods to differentiate strains (or subtypes) of $L$. monocytogenes has important epidemiological applications: rapid, precise, and efficient foodborne listeriosis surveillance can minimize outbreaks and track sources of L. monocytogenes contamination throughout the food system. Moreover, molecular subtyping in listeriosis surveillance would allow the detection of clusters of cases that could be erroneously considered as sporadic cases.

The association between a particular pulsotype and specific virulence traits is another possibility offered by molecular subtyping [11].

The study presented here was initiated in 2003 , with a view to support the establishment of an integrated, food chain surveillance system in Portugal. Ninety-five clinical Portuguese isolates recovered from apparently sporadic cases of listeriosis were collected, identified, biotyped by cadmium and arsenic sensitivity, geno-serotyped, and typed by DNA macrorestriction pulsed-field gel electrophoresis (PFGE). The aim was to obtain epidemiological data on cases of listeriosis in the country and to construct a molecular type database of clinical strains of L. monocytogenes.

\section{Materials and methods}

Collection of L. monocytogenes strains and related data to listeriosis episodes

A total of 95 L. monocytogenes strains isolated between 1994 and 2007 were collected from major Portuguese hospitals. The case definition of listeriosis employed was that contained in Commission Decision 2002/253/CE [12]. Thus, on isolation of L. monocytogenes from a hospitalized patient with a clinical compatible illness, each strain was considered as a single case of listeriosis. A case was defined as maternal/neonatal $(\mathrm{MN})$ in the following situations: infected pregnant woman, miscarriage, stillbirth, or newborn less than one month of age. Only one strain was kept when the pathogen was isolated from both the pregnant woman and her newborn child. If a case did not apply to any of these, it was considered as non-maternal/ neonatal (non-MN). Information regarding gender and age of the patient, the tissue or fluid origin where the bacteria was isolated, and the year of isolation was reported when available.

Confirmed isolates of L. monocytogenes were stored in Tryptic Soy Broth with $30 \%(\mathrm{v} / \mathrm{v})$ glycerol at $-80^{\circ} \mathrm{C}$ in the culture collection of the Escola Superior de Biotecnologia.
Biotyping by arsenic and cadmium sensitivity

The characterization of their differential resistance to arsenic and cadmium was performed in Isosensitest agar plates (ISA, Oxoid, Hampshire, UK) containing $500 \mu \mathrm{g} / \mathrm{ml}$ sodium arsenite (Merck, Darmstadt, Germany) or $75 \mu \mathrm{g} / \mathrm{ml}$ cadmium chloride (Merck) [13, 14].

\section{Geno-serotyping or PCR grouping}

Geno-serotyping was determined by PCR grouping with a multiplex PCR as described by Doumith et al. [9] using primers targeting fragments of genes lmo0737, ORF2819, ORF 2110, Imo1118, and prs (MWG-Biotech, Muenchenstein, Switzerland). PCR was performed in an Eppendorf thermocycler (Eppendorf, Hamburg, Germany) and PCR products were resolved on a $2 \%$ agarose gel containing $0.5 \mu \mathrm{g} / \mathrm{ml}$ of ethidium bromide (Eurobio, Courtaboeuf, France) and visualized and photographed under a UV transilluminator (Bio-Rad Gel Doc 2000 ${ }^{\mathrm{TM}}$ imaging system, Bio-Rad Laboratories, Milan, Italy).

\section{Molecular characterization by PFGE}

The DNA macrorestriction was performed at the Centre National de Référence des Listeria, Institut Pasteur (Paris, France) with the restriction enzymes AscI (New England BioLabs, Massachusetts, Ipswich, USA) and ApaI (MBI Fermentas, Burlington, Canada), as previously described by Graves and Swaminathan [10].

The pulsotypes obtained were scanned and the computerized data were analyzed using BioNumerics software version 5.1 (Applied Maths, Kortrijk, Belgium). Bands automatically assigned by the computer were checked visually and corrected manually when necessary. A position tolerance of 1.5 was selected for each PFGE. Cluster analysis of the individual or combined PFGE pulsotypes was done by the unweighted pair group method with average linkages (UPGMA), using the Dice coefficient to analyze the similarities of the banding pulsotypes. The discriminatory power was determined by calculating the discrimination index $(D)$ based on Simpson's index of diversity, as described by Hunter and Gaston [15].

Two PFGE patterns were considered to be indistinguishable when their similarity was higher than $98 \%$ [16] and a capital letter was ascribed to AscI pulsotype; when strains were similar at more than $98 \%$ by $A s c$ I but not similar at more than $98 \%$ by $A p a \mathrm{I}$, they were denoted, in addition to the capital letters, by lowercase letters.

PFGE pulsotypes of indistinguishable Portuguese strains were compared with PFGE pulsotypes of the French PFGE database of the Centre National de Référence des Listeria 
using the BioNumerics software package as previously described in this section.

\section{Statistical analysis}

The statistical package used for the analyses was Stata v10.0 (StataCorp LP, College Station, TX, USA). The contingency table analysis was based on the Chi-square distribution (Pearson's Chi-square test).

\section{Results}

Data collected from human episodes

A total of 95 isolates from cases of listeriosis were collected from major Portuguese hospitals during the study period. From the information available on 81 cases (Table 1), 85.2 and $14.8 \%$ corresponded to non-MN infections and $\mathrm{MN}$ infections, respectively. For the 69 confirmed non-MN cases, strains were isolated from blood (58.0\%), from cerebrospinal fluid (34.8\%), and from other specimens $(7.2 \%)$. The clinical symptoms were not recorded for the majority of cases. The gender ratio (M/ F) of confirmed non-MN cases was 2.3. The mean age of non-MN cases with documented age was 60 years, with 38 cases $(55.9 \%)$ being equal or up to 60 years. The geographical distribution of listeriosis cases was difficult to define from our collected data in the absence of data on the residence of patients.

Biotyping results by arsenic and cadmium sensitivity

Four groupings of sensitivity to heavy metals were differentiated among the 95 isolates (Table 1), the $D$-value for this biotyping method being 0.667 . The predominant group - sensitive to both arsenic (As) and cadmium (Cd) contains 46 isolates (48.4\%). Twenty-four isolates were arsenic-sensitive and cadmium-resistant (25.3\%), 18 were resistant to arsenic and sensitive to cadmium (18.9\%), and seven were resistant to both heavy metals $(7.4 \%)$.

\section{PCR grouping results}

Three PCR groups were identified in the 95 L. monocytogenes isolates recovered from sporadic human listeriosis cases in Portugal. Most isolates were of PCR group IVb (including serotypes $4 \mathrm{~b}$ or $4 \mathrm{~d}$ or $4 \mathrm{e}) 71.6 \%(n=68): 17.9 \%(n=17)$ and $10.5 \%(n=10)$ of the isolates showed PCR group IIb (including serotypes $1 / 2 \mathrm{~b}$ or $3 \mathrm{~b}$ ) and PCR group IIa (including serotypes $1 / 2 \mathrm{a}$ or $3 \mathrm{a}$ ), respectively. The $D$-value obtained from these PCR grouping results was 0.449 .
Combining the results from PCR grouping and sensitivity to heavy metals, it was possible to segregate the 95 isolates into nine groups $(D=0.831)$. Two groups predominate: PCR group IVb, As- and Cd-sensitive (30.5\%); PCR group IIb, As-resistant and Cd-sensitive (17.9\%).

\section{PFGE results}

PFGE typing revealed a total of $39 A s c \mathrm{I}$ and $50 \mathrm{ApaI}$ macrorestriction types among clinical isolates, distinguished by one or more band differences ranging in molecular size from 50 to $500 \mathrm{~kb}$ (Fig. 1). Combined analyses of $A s c \mathrm{I}$ and $A p a \mathrm{I}$ PFGE data yielded a total of 58 PFGE pulsotypes, with $D=0.966$ for $A s c \mathrm{I}, D=0.979$ for $A p a \mathrm{I}$, and $D=0.986$ for combined AscI and ApaI.

Comparisons based on such combined patterns are reported in Table 1 and the corresponding dendrogram in Fig. 1.

Pulsotypes DD, EE, FF, G, HHb, Jb, KKa, La, LLa, $\mathrm{LLb}, \mathrm{Me}, \mathrm{MM}, \mathrm{Pb}, \mathrm{S}, \mathrm{T}, \mathrm{U}, \mathrm{V}$, and Ya contained two or more strains which remained indistinguishable from each other. Only the pulsotypes FF, LLb, MM, Pb, T, U, V, and Ya contained indistinguishable strains by $\mathrm{Cd}$ and $\mathrm{As}$ sensitivity.

Pulsotypes DD, EE, FF, HHb, Jb, KKa, La, LLa, LLb, $\mathrm{Me}, \mathrm{MM}, \mathrm{Pb}, \mathrm{S}$, and $\mathrm{T}$ were constituted of L. monocytogenes isolates from geno-serotype $\mathrm{IVb}$, pulsotypes $\mathrm{U}, \mathrm{V}$, and Ya of geno-serotype IIb, and pulsotype G of geno-serotype IIa.

While strains of pulsotypes DD, FF, HHb, La, LLa, LLb, $\mathrm{MM}, \mathrm{Pb}, \mathrm{S}, \mathrm{T}, \mathrm{V}$, and $\mathrm{Ya}$ were recovered from different years and geographical distribution, pulsotypes EE, G, Jb, $\mathrm{KKa}, \mathrm{Me}$, and $\mathrm{U}$ were related in time or geographical distribution. Two pulsotypes ( $\mathrm{U}$ and $\mathrm{KKa}$ ) have six indistinguishable strains. Three pulsotype $U$ strains were isolated in the Lisbon region, respectively in February 2004, August 2004, and October 2004, with the same Cd and As sensitivity. Two pulsotype KKa strains having the same $\mathrm{Cd}$ and As sensitivity were isolated in March and in April 2004.

Pulsotype G contained two indistinguishable strains but with distinct $\mathrm{Cd}$ and As sensitivity isolated in February and May 2006 in different towns.

Concerning pulsotype EE, two indistinguishable strains but with distinct $\mathrm{Cd}$ and As sensitivity were isolated in different hospitals in Porto in 2007 six months apart, from a woman and a newborn child.

The pulsotype $\mathrm{Jb}$ contained three indistinguishable strains isolated in Lisbon in March 2006, September 2006, and June 2007. Pulsotype Me is constituted by four indistinguishable strains, three of which were isolated in the Porto region over a period of one year. 
Table 1 Listeria monocytogenes strains used in this study and collected epidemiological data concerning the cases with which they were associated

\begin{tabular}{|c|c|c|c|c|c|c|c|c|}
\hline \multirow[t]{2}{*}{ Isolate } & \multirow[t]{2}{*}{ Hospital localization } & \multirow[t]{2}{*}{ Year of isolation } & \multirow[t]{2}{*}{ Clinical form ${ }^{\mathrm{a}}$} & \multirow{2}{*}{$\begin{array}{l}\text { Patient } \\
\text { gender/age }\end{array}$} & \multirow{2}{*}{$\begin{array}{l}\text { PCR group } \\
\text { (geno-serotype) }\end{array}$} & \multirow{2}{*}{$\begin{array}{l}\text { Susceptibility } \\
\text { to } \mathrm{As} / \mathrm{Cd}^{\mathrm{c}}\end{array}$} & \multicolumn{2}{|c|}{ PFGE combined type } \\
\hline & & & & & & & $\mathrm{U}$ & I \\
\hline 771 & Porto & 2003 & M & Un $/<1$ month & $\mathrm{IVb}$ & $\mathrm{S} / \mathrm{S}$ & & $\mathrm{S}$ \\
\hline 779 & Porto & 2000 & $\mathrm{NM}$ & $\mathrm{F} / 25$ & $\mathrm{IVb}$ & $\mathrm{S} / \mathrm{R}$ & $\mathrm{HHa}$ & \\
\hline 780 & Porto & 1996 & $\mathrm{NM}$ & $\mathrm{M} / \mathrm{Un}$ & $\mathrm{IVb}$ & $\mathrm{S} / \mathrm{S}$ & & $\mathrm{HHb}$ \\
\hline 781 & Porto & 2000 & M & Un $/<1$ month & $\mathrm{IVb}$ & $\mathrm{S} / \mathrm{R}$ & & $\mathrm{HHb}$ \\
\hline 783 & Porto & 1994 & M & Un $/<1$ month & $\mathrm{IVb}$ & $\mathrm{R} / \mathrm{S}$ & & $\mathrm{S}$ \\
\hline 784 & Porto & 1999 & M & Un $/<1$ month & $\mathrm{IVb}$ & $\mathrm{R} / \mathrm{R}$ & $\mathrm{Ma}$ & \\
\hline 856 & Chaves & 2003 & NM & $\mathrm{M} / 50$ & $\mathrm{IVb}$ & $\mathrm{R} / \mathrm{S}$ & $\mathrm{Ka}$ & \\
\hline 866 & Matosinhos & 2003 & $\mathrm{NM}$ & M / 67 & $\mathrm{IVb}$ & $\mathrm{S} / \mathrm{S}$ & $\mathrm{R}$ & \\
\hline 908 & Chaves & 2003 & $\mathrm{NM}$ & $\mathrm{M} / 48$ & $\mathrm{IVb}$ & $\mathrm{R} / \mathrm{R}$ & & $\mathrm{HHb}$ \\
\hline 999 & Coimbra & 2000 & $\mathrm{NM}$ & $\mathrm{M} / 48$ & $\mathrm{IIb}$ & $\mathrm{S} / \mathrm{S}$ & $\mathrm{Z}$ & \\
\hline 1001 & Porto & 2004 & $\mathrm{NM}$ & $M / 65$ & $\mathrm{IVb}$ & $\mathrm{R} / \mathrm{R}$ & & $\mathrm{KKa}$ \\
\hline 1002 & Almada & 1998 & $\mathrm{NM}$ & $\mathrm{M} / 54$ & $\mathrm{IIb}$ & $\mathrm{S} / \mathrm{S}$ & $\mathrm{NN}$ & \\
\hline 1003 & Almada & 2003 & $\mathrm{NM}$ & $\mathrm{F} / 85$ & $\mathrm{IVb}$ & $\mathrm{S} / \mathrm{S}$ & & $\mathrm{LLa}$ \\
\hline 1037 & Chaves & 2004 & $\mathrm{NM}$ & $\mathrm{F} / 74$ & $\mathrm{IVb}$ & $\mathrm{S} / \mathrm{S}$ & $\mathrm{Pd}$ & \\
\hline 1059 & Matosinhos & 2004 & $\mathrm{NM}$ & $\mathrm{F} / 73$ & $\mathrm{IVb}$ & $S / R$ & & $\mathrm{HHb}$ \\
\hline 1062 & Lisbon & 2004 & $\mathrm{NM}$ & $\mathrm{M} / 54$ & $\mathrm{IIb}$ & $\mathrm{S} / \mathrm{S}$ & $\mathrm{W}$ & \\
\hline 1063 & Lisbon & 2002 & $\mathrm{NM}$ & $\mathrm{M} / \mathrm{Un}$ & $\mathrm{IVb}$ & $\mathrm{S} / \mathrm{S}$ & & $\mathrm{FF}$ \\
\hline 1065 & Lisbon & 2004 & $\mathrm{NM}$ & $\mathrm{M} / \mathrm{Un}$ & $\mathrm{IIb}$ & $\mathrm{S} / \mathrm{S}$ & & $\mathrm{U}$ \\
\hline 1198 & Matosinhos & 2004 & $\mathrm{NM}$ & $\mathrm{M} / 74$ & IIa & $\mathrm{S} / \mathrm{S}$ & $\mathrm{H}$ & \\
\hline 1239 & Matosinhos & 2004 & $\mathrm{M}$ & Un $/<1$ month & $\mathrm{IVb}$ & $\mathrm{R} / \mathrm{S}$ & BB & \\
\hline 1240 & Almada & 2004 & M & $\mathrm{F} /<1$ month & $\mathrm{IIb}$ & $\mathrm{S} / \mathrm{S}$ & & $\mathrm{U}$ \\
\hline 1241 & Coimbra & 2004 & $\mathrm{NM}$ & $\mathrm{M} / 44$ & $\mathrm{IVb}$ & $\mathrm{R} / \mathrm{R}$ & & $\mathrm{KKa}$ \\
\hline 1242 & Lisbon & 2004 & $\mathrm{NM}$ & $\mathrm{M} / 57$ & $\mathrm{IIb}$ & $\mathrm{S} / \mathrm{S}$ & & $\mathrm{U}$ \\
\hline 1244 & Lisbon & 2004 & $\mathrm{NM}$ & $\mathrm{M} / 46$ & $\mathrm{IVb}$ & $\mathrm{R} / \mathrm{S}$ & $\mathrm{N}$ & \\
\hline 1348 & Almada & 2005 & $\mathrm{NM}$ & $\mathrm{F} / 77$ & $\mathrm{IIb}$ & $\mathrm{S} / \mathrm{S}$ & & $\mathrm{U}$ \\
\hline $1383 \mathrm{~A}$ & Lisbon & 2005 & $\mathrm{NM}$ & $\mathrm{M} / 40$ & $\mathrm{IVb}$ & $\mathrm{R} / \mathrm{R}$ & $\mathrm{JJ}$ & \\
\hline 1541 & Coimbra & 2000 & $\mathrm{NM}$ & Un / Un & $\mathrm{IVb}$ & $\mathrm{S} / \mathrm{S}$ & & LLb \\
\hline 1542 & Coimbra & 2000 & $\mathrm{NM}$ & Un / Un & $\mathrm{IVb}$ & $\mathrm{S} / \mathrm{R}$ & $\mathrm{HHc}$ & \\
\hline 1543 & Coimbra & 2000 & $\mathrm{NM}$ & Un / Un & $\mathrm{IVb}$ & $\mathrm{S} / \mathrm{S}$ & $\mathrm{Ja}$ & \\
\hline 1544 & Coimbra & 2000 & $\mathrm{NM}$ & Un / Un & $\mathrm{IVb}$ & $\mathrm{S} / \mathrm{S}$ & & DD \\
\hline 1545 & Coimbra & 2003 & M & $\mathrm{F} / 31$ & $\mathrm{IIb}$ & $\mathrm{S} / \mathrm{S}$ & & Ya \\
\hline 1546 & Coimbra & 2001 & $\mathrm{NM}$ & $\mathrm{M} / 41$ & $\mathrm{IVb}$ & $\mathrm{S} / \mathrm{S}$ & $\mathrm{Pc}$ & \\
\hline 1547 & Coimbra & 2001 & $\mathrm{NM}$ & $\mathrm{M} / 85$ & $\mathrm{IIb}$ & $\mathrm{S} / \mathrm{S}$ & & $\mathrm{U}$ \\
\hline 1548 & Coimbra & 1999 & $\mathrm{NM}$ & Un / Un & $\mathrm{IVb}$ & $\mathrm{S} / \mathrm{S}$ & & $\mathrm{KKa}$ \\
\hline 1549 & Coimbra & 1999 & $\mathrm{NM}$ & Un / Un & $\mathrm{IVb}$ & $\mathrm{S} / \mathrm{R}$ & & MM \\
\hline 1550 & Coimbra & 1997 & $\mathrm{NM}$ & Un / Un & $\mathrm{IVb}$ & $\mathrm{S} / \mathrm{S}$ & & LLc \\
\hline 1551 & Coimbra & 1997 & $\mathrm{NM}$ & Un / Un & $\mathrm{IVb}$ & $\mathrm{S} / \mathrm{S}$ & & $\mathrm{T}$ \\
\hline 1552 & Coimbra & 1997 & $\mathrm{NM}$ & Un / Un & $\mathrm{IVb}$ & $\mathrm{S} / \mathrm{S}$ & $\mathrm{Md}$ & \\
\hline 1553 & Coimbra & 1998 & $\mathrm{NM}$ & Un / Un & $\mathrm{IVb}$ & $\mathrm{R} / \mathrm{S}$ & & $\mathrm{La}$ \\
\hline 1554 & Coimbra & 1999 & $\mathrm{NM}$ & Un / Un & $\mathrm{IVb}$ & $\mathrm{S} / \mathrm{R}$ & & $\mathrm{KKa}$ \\
\hline 1555 & Coimbra & 2003 & $\mathrm{NM}$ & $\mathrm{F} / 74$ & $\mathrm{IVb}$ & $\mathrm{S} / \mathrm{S}$ & & $\mathrm{T}$ \\
\hline 1556 & Coimbra & 2005 & $\mathrm{NM}$ & $\mathrm{F} / 56$ & $\mathrm{IVb}$ & $\mathrm{S} / \mathrm{S}$ & & $\mathrm{Pb}$ \\
\hline $1562 / 1$ & Lisbon & 2005 & $\mathrm{NM}$ & $\mathrm{M} / 81$ & $\mathrm{IIb}$ & $\mathrm{S} / \mathrm{R}$ & $\mathrm{Yb}$ & \\
\hline $1621 / 1$ & Lisbon & 2005 & $\mathrm{NM}$ & $\mathrm{F} / 72$ & $\mathrm{IVb}$ & $\mathrm{S} / \mathrm{S}$ & & LLb \\
\hline 1761 & Coimbra & 2005 & $\mathrm{NM}$ & M / 57 & $\mathrm{IIb}$ & $\mathrm{S} / \mathrm{R}$ & A & \\
\hline 1762 & Coimbra & 2005 & M & $\mathrm{F} / 33$ & $\mathrm{IIb}$ & $\mathrm{S} / \mathrm{R}$ & & $\mathrm{V}$ \\
\hline 1763 & Coimbra & 2005 & $\mathrm{NM}$ & $\mathrm{M} / 51$ & $\mathrm{IVb}$ & $\mathrm{R} / \mathrm{S}$ & Mf & \\
\hline 1764 & Coimbra & 2005 & $\mathrm{M}$ & Un $/<1$ month & $\mathrm{IVb}$ & $\mathrm{S} / \mathrm{R}$ & $\mathrm{KKc}$ & \\
\hline 1765 & Coimbra & 2005 & M & $\mathrm{F} / 31$ & $\mathrm{IVb}$ & $\mathrm{S} / \mathrm{S}$ & $\mathrm{Kb}$ & \\
\hline 1766 & Braga & 2005 & $\mathrm{NM}$ & $\mathrm{F} / 4$ months & $\mathrm{IVb}$ & $\mathrm{S} / \mathrm{R}$ & & $\mathrm{Me}$ \\
\hline 1767 & Braga & 2005 & $\mathrm{NM}$ & $\mathrm{F} / 46$ & $\mathrm{IVb}$ & $\mathrm{S} / \mathrm{R}$ & & $\mathrm{KKa}$ \\
\hline 1768 & Matosinhos & 2006 & $\mathrm{M}$ & $\mathrm{F} / 31$ & $\mathrm{IVb}$ & $\mathrm{R} / \mathrm{S}$ & & $\mathrm{Me}$ \\
\hline 1792 & Lisbon & 2006 & $\mathrm{NM}$ & $\mathrm{M} / 34$ & $\mathrm{IVb}$ & $\mathrm{R} / \mathrm{S}$ & & $\mathrm{Jb}$ \\
\hline 1796 & Lisbon & 2006 & NM & $\mathrm{F} / 80$ & $\mathrm{IIb}$ & $\mathrm{S} / \mathrm{R}$ & & $\mathrm{V}$ \\
\hline
\end{tabular}


Table 1 (continued)

\begin{tabular}{|c|c|c|c|c|c|c|c|c|}
\hline \multirow[t]{2}{*}{ Isolate } & \multirow[t]{2}{*}{ Hospital localization } & \multirow[t]{2}{*}{ Year of isolation } & \multirow[t]{2}{*}{ Clinical form ${ }^{\mathrm{a}}$} & \multirow{2}{*}{$\begin{array}{l}\text { Patient } \\
\text { gender/age }\end{array}$} & \multirow{2}{*}{$\begin{array}{l}\text { PCR group } \\
\text { (geno-serotype) }\end{array}$} & \multirow{2}{*}{$\begin{array}{l}\text { Susceptibility } \\
\text { to } \mathrm{As} / \mathrm{Cd}^{\mathrm{c}}\end{array}$} & \multicolumn{2}{|c|}{ PFGE combined type } \\
\hline & & & & & & & $\mathrm{U}$ & I \\
\hline 1803 & Matosinhos & 2006 & NM & $\mathrm{M} / 77$ & $\mathrm{IVb}$ & $\mathrm{R} / \mathrm{S}$ & $\mathrm{Lb}$ & \\
\hline $1807 / 1$ & Lisboan & 2006 & $\mathrm{NM}$ & $\mathrm{F} / 68$ & $\mathrm{IVb}$ & $\mathrm{S} / \mathrm{R}$ & & $\mathrm{KKa}$ \\
\hline 1878 & Lisbon & 2006 & NM & $\mathrm{M} / 70$ & $\mathrm{IVb}$ & $\mathrm{S} / \mathrm{S}$ & & $\mathrm{LLa}$ \\
\hline 1879 & Lisbon & 2006 & $\mathrm{NM}$ & $\mathrm{M} / 58$ & $\mathrm{IVb}$ & $\mathrm{R} / \mathrm{R}$ & & $\mathrm{Jb}$ \\
\hline 1880 & Porto & 2006 & $\mathrm{NM}$ & $\mathrm{M} / 78$ & IIa & $\mathrm{S} / \mathrm{S}$ & $\mathrm{C}$ & \\
\hline 1891 & Braga & 2006 & NM & $\mathrm{M} / 60$ & IIa & $\mathrm{S} / \mathrm{S}$ & F & \\
\hline 1999 & Lisbon & 2006 & $\mathrm{NM}$ & $\mathrm{M} / 70$ & $\mathrm{IVb}$ & $\mathrm{S} / \mathrm{S}$ & & $\mathrm{KKb}$ \\
\hline 2029 & Lisbon & 2007 & $\mathrm{NM}$ & $\mathrm{F} / 81$ & IIa & $\mathrm{S} / \mathrm{R}$ & $\mathrm{D}$ & \\
\hline 2064 & Matosinhos & 2006 & NM & $M / 63$ & $\mathrm{IVb}$ & $\mathrm{R} / \mathrm{S}$ & & $\mathrm{La}$ \\
\hline 2065 & Matosinhos & 2007 & $\mathrm{NM}$ & $\mathrm{F} / 79$ & $\mathrm{IIb}$ & $\mathrm{S} / \mathrm{R}$ & $\mathrm{X}$ & \\
\hline 2074 & Porto & 2007 & M & Un $/<1$ month & $\mathrm{IVb}$ & $\mathrm{S} / \mathrm{S}$ & & $\mathrm{EE}$ \\
\hline 2076 & Lisbon & 2007 & NM & $\mathrm{F} / 50$ & $\mathrm{IIb}$ & $\mathrm{S} / \mathrm{S}$ & & $\mathrm{Ya}$ \\
\hline 2085 & Lisbon & 2007 & NM & $\mathrm{F} / 73$ & $\mathrm{IVb}$ & $\mathrm{S} / \mathrm{S}$ & $\mathrm{Pa}$ & \\
\hline 2086 & Matosinhos & 2007 & $\mathrm{NM}$ & $\mathrm{M} / 84$ & IIa & $\mathrm{S} / \mathrm{S}$ & B & \\
\hline 2087 & Lisbon & 2007 & NM & $\mathrm{M} / 50$ & $\mathrm{IVb}$ & $\mathrm{S} / \mathrm{R}$ & $\mathrm{Mc}$ & \\
\hline 2088 & Porto & 2006 & $\mathrm{NM}$ & M / 77 & $\mathrm{IVb}$ & $\mathrm{S} / \mathrm{S}$ & & $\mathrm{FF}$ \\
\hline 2090 & Porto & 2006 & $\mathrm{NM}$ & $\mathrm{M} / 54$ & $\mathrm{IVb}$ & $\mathrm{S} / \mathrm{R}$ & & $\mathrm{Me}$ \\
\hline 2091 & Porto & 2006 & NM & M / 61 & IIa & $\mathrm{R} / \mathrm{S}$ & & $\mathrm{G}$ \\
\hline 2092 & Braga & 2007 & $\mathrm{NM}$ & $\mathrm{M} / 78$ & $\mathrm{IVb}$ & $\mathrm{R} / \mathrm{S}$ & $\mathrm{Mb}$ & \\
\hline 2093 & Braga & 2007 & NM & $\mathrm{M} / 77$ & $\mathrm{IVb}$ & $\mathrm{R} / \mathrm{R}$ & GG & \\
\hline 2094 & Funchal & 2007 & $\mathrm{NM}$ & $\mathrm{F} / 53$ & $\mathrm{IVb}$ & $\mathrm{S} / \mathrm{S}$ & & $\mathrm{FF}$ \\
\hline 2095 & Almada & 2007 & NM & $\mathrm{F} / 88$ & $\mathrm{IIb}$ & $\mathrm{S} / \mathrm{S}$ & Q & \\
\hline 2096 & Porto & 2005 & NM & $\mathrm{M} / 40$ & $\mathrm{IVb}$ & $\mathrm{S} / \mathrm{S}$ & & $\mathrm{La}$ \\
\hline 2097 & Coimbra & 2006 & $\mathrm{NM}$ & $\mathrm{F} / 81$ & $\mathrm{IVb}$ & $\mathrm{R} / \mathrm{S}$ & & DD \\
\hline 2098 & Coimbra & 2006 & $\mathrm{NM}$ & $\mathrm{M} / 83$ & $\mathrm{IIb}$ & $\mathrm{S} / \mathrm{R}$ & AA & \\
\hline 2099 & Coimbra & 2006 & NM & $\mathrm{M} / 53$ & IIa & $\mathrm{S} / \mathrm{S}$ & & G \\
\hline 2100 & Coimbra & 2006 & NM & $\mathrm{F} / 70$ & $\mathrm{IVb}$ & $\mathrm{S} / \mathrm{S}$ & & $\mathrm{FF}$ \\
\hline 2101 & Coimbra & 2007 & $\mathrm{NM}$ & M / 66 & $\mathrm{IVb}$ & $\mathrm{S} / \mathrm{S}$ & II & \\
\hline 2102 & Lisbon & 2007 & NM & $\mathrm{M} / 74$ & $\mathrm{IVb}$ & $\mathrm{R} / \mathrm{S}$ & $\mathrm{Jb}$ & \\
\hline 2103 & Lisbon & 2007 & $\mathrm{NM}$ & M / 19 & IIa & $\mathrm{S} / \mathrm{S}$ & $\mathrm{E}$ & \\
\hline 2104 & Coimbra & 2006 & $\mathrm{NM}$ & $\mathrm{F} / 68$ & $\mathrm{IVb}$ & $\mathrm{S} / \mathrm{R}$ & & MM \\
\hline 2105 & Coimbra & 2007 & NM & $\mathrm{M} / 43$ & $\mathrm{IVb}$ & $\mathrm{S} / \mathrm{R}$ & & MM \\
\hline 2117 & Braga & 2007 & $\mathrm{NM}$ & $\mathrm{M} / 76$ & IVa & $\mathrm{S} / \mathrm{S}$ & $\mathrm{CC}$ & \\
\hline 2121 & Braga & 2007 & NM & $\mathrm{M} / 34$ & $\mathrm{IIb}$ & $\mathrm{S} / \mathrm{S}$ & & $\mathrm{U}$ \\
\hline 2122 & Lisbon & 2007 & $\mathrm{NM}$ & $\mathrm{M} / 88$ & $\mathrm{IVb}$ & $\mathrm{S} / \mathrm{S}$ & & $\mathrm{Pb}$ \\
\hline 2125 & Funchal & 2007 & $\mathrm{NM}$ & $\mathrm{M} / 58$ & $\mathrm{IVb}$ & $\mathrm{R} / \mathrm{S}$ & & $\mathrm{EE}$ \\
\hline 2135 & Lisbon & 2007 & NM & $\mathrm{M} / 52$ & IIa & $\mathrm{S} / \mathrm{R}$ & I & \\
\hline 2252 & Porto & 2007 & $\mathrm{NM}$ & $\mathrm{M} / 50$ & $\mathrm{IVb}$ & $\mathrm{R} / \mathrm{S}$ & & $\mathrm{Me}$ \\
\hline 2253 & Porto & 2007 & $\mathrm{NM}$ & M / 60 & $\mathrm{IVb}$ & $\mathrm{R} / \mathrm{S}$ & & DD \\
\hline 2254 & Porto & 2007 & $\mathrm{NM}$ & M / 69 & $\mathrm{IVb}$ & $\mathrm{S} / \mathrm{S}$ & $\mathrm{O}$ & \\
\hline 2255 & Porto & 2007 & NM & $\mathrm{F} / 26$ & IVa & $\mathrm{R} / \mathrm{S}$ & & $\mathrm{EE}$ \\
\hline
\end{tabular}

Strains not similar at more than $98 \%$ by PFGE typing with AscI were addressed as unique, ascribed a capital letter, and in the column U (unique). Strains similar at equal to or more than $98 \%$ from at least one other isolate were listed in column I (indistinguishable) and were further typed with ApaI. Strains similar at more than $98 \%$ by $A s c \mathrm{I}$ but not similar at more than $98 \%$ by $A p a$ I were denoted, in addition to the capital letters, by lowercase letters. So, a combined type from PCR grouping as well as from PFGE typing was determined. Strains distinguishable by their PCR grouping or strains not similar at more than $98 \%$ by PFGE typing with AscI and ApaI were again listed as unique (U). Strains not distinguishable by their PCR grouping or strains similar at equal to or more than $98 \%$ by PFGE typing with $A s c$ I and/or ApaI were again listed as indistinguishable (I)

${ }^{\text {a }}$ M: maternal/neonatal; NM, non-maternal/neonatal

${ }^{\mathrm{b}}$ Un: unknown

${ }^{\mathrm{c}} \mathrm{R}$ : resistant; S: sensitive 


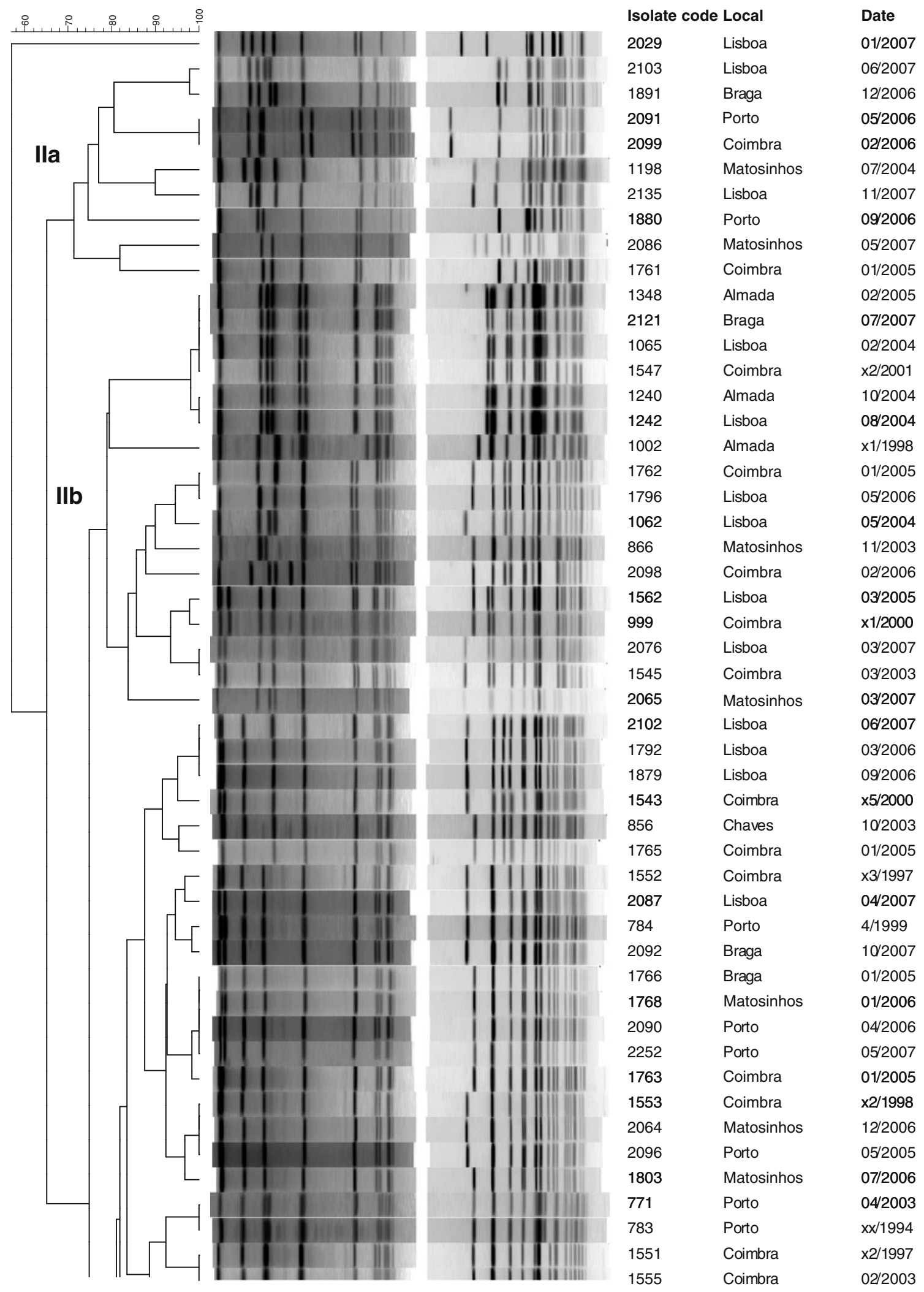

Fig. 1 Dendrogram for Listeria monocytogenes pulsotypes of all 95 isolates obtained from clinical cases, Portugal, 1994-2007 


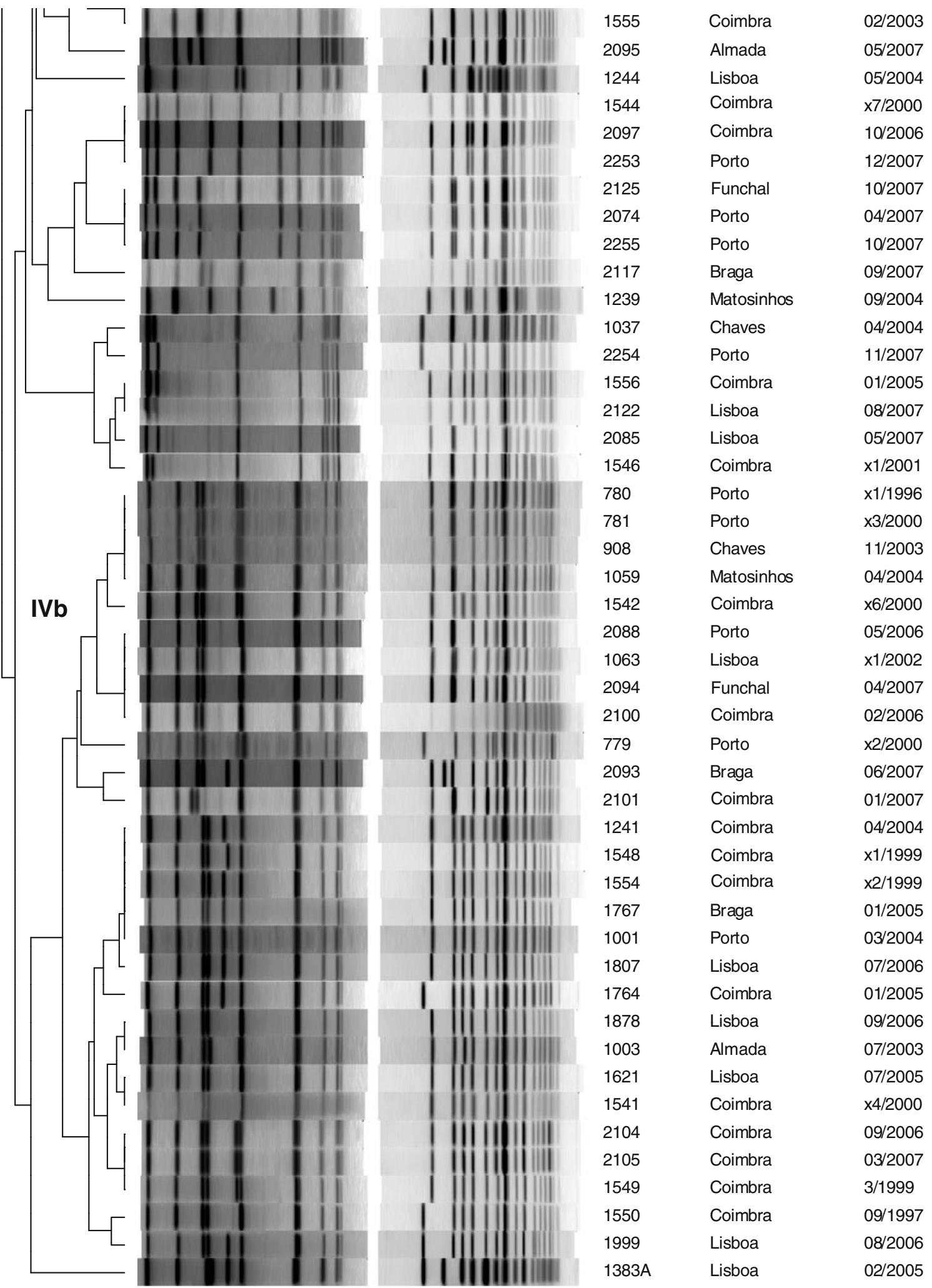

Fig. 1 (continued) 
Comparison with the PFGE pulsotypes database of French human strains underlined that pulsotypes $\mathrm{Me}, \mathrm{Jb}, \mathrm{Pb}, \mathrm{HHb}$, FF, MM, and LLb have been previously described in human clusters in France and are part of the main pulsotypes from human strains observed in France. Pulsotypes MM and LLb are indistinguishable from pulsotypes associated with the main outbreak of listeriosis in France in 1992. The main pulsotypes observed in Portugal, $\mathrm{U}$ and $\mathrm{KKa}$, have not been recorded for French human cases.

Combination of biotyping, PCR grouping, and PFGE results

The highest $D$-value, 0.994 , and, thus, the greatest number of distinguishable types (72) was obtained when the results of multiplex PCR, sensitivity to heavy metals, and analysis of $A s c \mathrm{I}$ and $A p a \mathrm{I}$ were combined.

\section{Discussion}

The 2007 Annual Community Summary Report by the European Food Safety Authority (EFSA) and the European Centre for Disease Prevention and Control (ECDC) established an overall incidence rate of listeriosis in Europe of 3 cases per million inhabitants, with a range of $0-11$ cases per million inhabitants. The results presented here estimated an incidence rate of listeriosis in Portugal, based on information from voluntary reporting, of 2.3 cases per million inhabitants for the year 2007, which is comparable to the reported incidence in Austria, Estonia, Latvia, Slovakia, and Slovenia [17]. In Portugal's only neighboring country, Spain, the incidence for 2007 was similar to that described in this study and was also based on the voluntary reporting of cases to the National Reference Laboratory. However, it has been demonstrated that the implementation of an active surveillance system for listeriosis in Navarra, a region of northern Spain, led to an incidence for this region similar to that described in countries with mandatory notification of listeriosis, such as France, Denmark, and the Netherlands [18]. An apparent increase in the numbers of cases of listeriosis in recent years, observed in some other countries, e.g., Denmark, Finland, France, Germany, the Netherlands, Switzerland, and United Kingdom $[3,4]$, was also observed in Portugal. These results indicate that voluntary reporting underestimates the occurrence of listeriosis in a country, and that the $\mathrm{EC}$ requirement for the statutory reporting of listeriosis will probably lead to many more cases being uncovered. A statutory reporting and investigation system in Portugal will inevitably take some time to establish, and it is recommended that, in parallel, a surveillance system for the pathogen in typical foods should be established, with the capability for in-depth characterization of isolates for comparisons of clinical and food strains.
The majority of patients with listeriosis in Portugal were people older than 60 years with bacteremia, as recorded in some other countries, including France and United Kingdom [19, 20]. Most of these cases were caused by PCR group IVb strains, which are generally associated with epidemic clones [21]. As in other countries [18, 20], serogroup $1 / 2$ a became more common than serogroup $1 / 2 b$ in 2006 and 2007.

The results of our study confirm that PCR grouping alone has the lowest discriminatory power but, when combined with PFGE AscI and ApaI, it becomes the most powerful of all [22]. Nevertheless, during outbreaks, the screening method of strains based on PCR grouping rather than classical serotyping is useful. Biotyping by $\mathrm{As}$ and $\mathrm{Cd}$ sensitivity gave additional information data but not enough to consider the use of this method in a routine system of microbiological surveillance. As observed in Belgium [23], an increase in strains resistant to $\mathrm{Cd}$ but sensitive to As was verified.

In our study, it was possible to link sporadic cases on six occasions related in time and geographical distributions; thus, a common source of contamination could be suspected. This suspicion underlined the need to characterize food and environmental strains at the same time as human strains at a national level in order to trace sources of contamination and to detect and confirm related cases.

The finding that combined pulsotypes of Portuguese clinical isolates have already been described in human clusters, and even outbreaks, in France can be explained by both human travel and food commerce. It can be argued that there is, at least, a European distribution of clinical strains that necessitates European tracing, such as a PulseNet initiative for the continent. Nevertheless, combined pulsotypes $\mathrm{U}$ and $\mathrm{KKa}$ have been detected in Portugal but not in France, which suggests that indigenous clones of L. monocytogenes may exist in the country which might, at some time, become epidemic clones.

The possible epidemiological links with human strains isolated in other countries underlines the need for an active surveillance system of listeriosis in Portugal harmonized with other national systems. As the French National Surveillance System [20, 24], this should be based on the obligatory notification of human cases of listeriosis and epidemiological data collection, on the basis of European regulation EC 178/2002 (obligatory notification of unsatisfactory food according to microbiological criteria defined by European regulation), and also on the dispatch of isolates to a national reference laboratory for typing.

Acknowledgments This work was supported by FCT project PTDC/AGR-ALI/64662/2006, "Listeria monocytogenes in foods: contributing data for risk assessment".

Gonçalo Almeida was the recipient of a Fundação Calouste Gulbenkian short visit grant 96780 . 
Grateful acknowledgment goes to Laboratoire des Listeria, Centre National de Référence des Listeria, WHO Collaborating Center for Foodborne Listeriosis, Institut Pasteur for accepting Gonçalo Almeida and PFGE typing the strains of this work.

Members of the Research Team Ana Florinda (Centro Hospitalar de Coimbra), Graça Ribeiro and Luisa Boaventura (Hospitais da Universidade de Coimbra), Teresa Afonso (Hospital Central do Funchal do Serviço de Saúde da Região Autónoma da Madeira), Helena Peres, Teresa Pina, and Maria José Silvestre (Hospital Curry Cabral, Lisboa), Maria Dolores Pinheiro (Hospital de São João, Porto), Maria Alberta Faustino and Maria Carmen Iglesias (Hospital de São Marcos, Braga), José Diogo, Ana Rodrigues, and Isabel Nascimento (Hospital Garcia da Horta, Almada), Fernanda Bessa and Elmano Ramalheira (Hospital Infante D. Pedro-Aveiro, E.P.E.), João Lago (Hospital Militar de Belém, Lisboa), Maria Antónia Read (Hospital Pedro Hispano, Matosinhos), Lurdes Monteiro, Luís Marques Lito, and J. Melo Cristino (Hospital Santa Maria, Lisboa), Maria Helena Ramos (Hospital Santo António, Porto), Maria Augusta Guimarães (Instituto Português de Oncologia, Porto).

\section{References}

1. Wing EJ, Gregory SH (2002) Listeria monocytogenes: clinical and experimental update. J Infect Dis 185:S18-S24

2. Almeida GN, Gibbs PA, Hogg TA, Teixeira PC (2006) Listeriosis in Portugal: an existing but under reported infection. BMC Infect Dis 6:153

3. Doorduyn Y, de Jager CM, van der Zwaluw WK, Wannet WJ, van der Ende A, Spanjaard L, van Duynhoven YT (2006) First results of the active surveillance of Listeria monocytogenes infections in the Netherlands reveal higher than expected incidence. Euro Surveill 11(4):E060420.4

4. Goulet V, Hedberg C, Le Monnier A, de Valk H (2008) Increasing incidence of listeriosis in France and other European countries. Emerg Infect Dis 14(5):734-740

5. Bille J, Blanc DS, Schmid H, Boubaker K, Baumgartner A, Siegrist HH, Tritten ML, Lienhard R, Berner D, Anderau R, Treboux M, Ducommun JM, Malinverni R, Genné D, Erard PH, Waespi U (2006) Outbreak of human listeriosis associated with tomme cheese in northwest Switzerland, 2005. Euro Surveill 11(6):91-93

6. Dawson SJ, Evans MR, Willby D, Bardwell J, Chamberlain N, Lewis DA (2006) Listeria outbreak associated with sandwich consumption from a hospital retail shop, United Kingdom. Euro Surveill 11(6):89-91

7. Vít M, Olejník R, Dlhý J, Karpíšková R, Cástková J, Príkazský V, Príkazská M, Beneš C, Petráš P (2007) Outbreak of listeriosis in the Czech Republic, late 2006 - preliminary report. Euro Surveill 12(6):E070208.1

8. Fretz R, Sagel U, Ruppitsch W, Pietzka AT, Stöger A, Huhulescu S, Heuberger S, Pichler J, Much P, Pfaff G, Stark K, Prager R, Flieger A, Feenstra O, Allerberger F (2010) Listeriosis outbreak caused by acid curd cheese 'Quargel', Austria and Germany 2009. Euro Surveill 15(5):pii=19477
9. Doumith M, Buchrieser C, Glaser P, Jacquet C, Martin P (2004) Differentiation of the major Listeria monocytogenes serovars by multiplex PCR. J Clin Microbiol 42:3819-3822

10. Graves LM, Swaminathan B (2001) PulseNet standardized protocol for subtyping Listeria monocytogenes by macrorestriction and pulsed-field gel electrophoresis. Int J Food Microbiol 65:55-62

11. Franciosa G, Tartaro S, Wedell-Neergaard C, Aureli P (2001) Characterization of Listeria monocytogenes strains involved in invasive and noninvasive listeriosis outbreaks by PCR-based fingerprinting techniques. Appl Environ Microbiol 67:1793-1799

12. European Commission (2002) Case definitions for reporting communicable disease to the community network. Commission decision No. 2119/98/EC of the European parliament and of the council. Official Journal of the European communities L86/44 (3.04.2002):52

13. Vaz-Velho M, Duarte G, McLauchlin J, Gibbs P (2001) Characterization of Listeria monocytogenes isolated from production lines of fresh and cold-smoked fish. J Appl Microbiol 91:556-562

14. McLauchlin J, Hampton MD, Shah S, Threlfall EJ, Wieneke AA, Curtis GDW (1997) Subtyping of Listeria monocytogenes on the basis of plasmid profiles and arsenic and cadmium susceptibility. $\mathrm{J}$ Appl Microbiol 83:381-388

15. Hunter PR, Gaston MA (1988) Numerical index of the discriminatory ability of typing systems: an application of Simpson's index of diversity. J Clin Microbiol 26:2465-2466

16. Tenover FC, Arbeit RD, Goering RV, Mickelsen PA, Murray BE, Persing DH, Swaminathan B (1995) Interpreting chromosomal DNA restriction patterns produced by pulsed-field gel electrophoresis: criteria for bacterial strain typing. J Clin Microbiol 33:2233-2239

17. European Food Safety Authority (EFSA) (2009) The community summary report on trends and sources of zoonoses and zoonotic agents in the European Union in 2007. The EFSA Journal 223. Available online at: http://www.efsa.europa.eu/en/scdocs/scdoc/ 223r.htm

18. Garrido V, Torroba L, García-Jalón I, Vitas A (2008) Surveillance of listeriosis in Navarre, Spain, 1995-2005-epidemiological patterns and characterisation of clinical and food isolates. Euro Surveill 13(49):pii $=19058$

19. Gillespie IA, McLauchlin J, Grant KA, Little CL, Mithani V, Penman C, Lane C, Regan M (2006) Changing pattern of human listeriosis, England and Wales, 2001-2004. Emerg Infect Dis 12 (9):1361-1366

20. Goulet V, Jacquet C, Martin P, Vaillant V, Laurent E, de Valk H (2006) Surveillance of human listeriosis in France, 2001-2003. Euro Surveill 11:79-81

21. Swaminathan B, Gerner-Smidt P (2007) The epidemiology of human listeriosis. Microbes Infect 9:1236-1243

22. Jacquet C, Catimel B, Brosch R, Buchrieser C, Dehaumont P, Goulet V, Lepoutre A, Veit P, Rocourt J (1995) Investigations related to the epidemic strain involved in the french listeriosis outbreak in 1992. Appl Environ Microbiol 61:2242-2246

23. Centre National de Reference des Listeria (2004) Rapport annuel souches de Listeria isolées en Belgique en 2004

24. Le Monnier A, Leclercq A (2009) Listeria et listériose: des animaux d'élevage à nos assiettes. Pathol Biol 57:17-22 